\title{
Análisis de la función pulmonar en trabajadores de minería mediante espirometría
}

\author{
Analysis of the pulmonary function in \\ mining workers by spirometry
}

Javier Eliécer Pereira Rodríguez; ${ }^{1}$ DeVi Geesel Peñaranda Flórez; ${ }^{2}$

Juan Camilo Quintero Gómez; ${ }^{3}$ Alfredo Cruz Morales; ${ }^{4}$ Daniela del Carmen luna Martínez. ${ }^{4}$

\section{Resumen}

INTRODUCCIÓN: la minería es una actividad comercial del sector industrial que tiene como objeto la explotación de suelos para la obtención de nuevos recursos. La ocupación de los trabajos de minería se ha asociado con el desarrollo de diferentes alteraciones a nivel respiratorio.

OBJETIVO: determinar el estado de la función pulmonar en trabajadores de minería mediante espirometría.

MATERIALES Y MÉTODOS: se llevó a cabo un estudio descriptivo de corte transversal con 848 trabajadores del sector de la minería. Se realizó la prueba de función pulmonar por espirometría y se registraron las mediciones siguiendo las recomendaciones de la American Thoracic Society (ATS).

RESULTADOS: $7(0,83 \%)$ casos de la población presentaron enfermedad pulmonar obstructiva crónica (EPOC) en grado leve y $1(0,12 \%)$ caso con obstrucción pulmonar grave. Adicionalmente, se encontraron $241(28,42 \%)$ casos de enfermedad con patrón restrictivo leve, $2(0,24 \%)$ con restricción moderada y $1(0,12 \%)$ con restricción pulmonar grave.

CONCLUSIONES: la probabilidad de enfermedad respiratoria de patrón restrictivo es mayor en la población minera. Los valores espirométricos son significativamente menores en sujetos con ocupación en el sector minero.

Palabras clave: función pulmonar, minería, espirometría.

\begin{abstract}
INTRODUCTION: Mining is a commercial activity of the industrial sector whose purpose is the exploitation of soils to obtain new resources. The occupation of mining works has been associated with the development of different alterations at the respiratory level.

OBJECTIVE: To determine the state of pulmonary function in mining workers using spirometry.

MATERIALS AND METHODS: A descriptive cross-sectional study was carried out with 848 workers from the mining sector. The pulmonary function test was performed by spirometry and the measurements were recorded following the recommendations of the American Thoracic Society (ATS).
\end{abstract}

Grupo de investigación Alétheia

${ }^{1}$ Fisioterapeuta, Especialista en Rehabilitación Cardiopulmonar, Maestrante en Ciencias de la Salud. Docente Cardiopulmonar, Universidad Tolteca. Puebla, México.

${ }^{2}$ Fisioterapeuta, Especialista en Neurorrehabilitación. Magíster en dificultades del aprendizaje. Consultorio Independiente Privado. Puebla, México.

${ }^{3}$ Estudiante de Fisioterapia. Universidad de Santander. Cúcuta, Colombia.

${ }^{4}$ Estudiante de Fisioterapia. Instituto Profesional en Terapias y Humanidades. Puebla, México.

Autor de correspondencia

Javier Pereira Rodríguez. Correo electrónico: jepr87@hotmail.com.

Recibido: 7/1/2019. Aceptado: 25/4/2019. 
RESULTS: $7(0.83 \%)$ cases of the population had mild chronic obstructive pulmonary disease (COPD) and $1(0.12 \%)$ case with severe pulmonary obstruction. On the other hand, $241(28.42 \%)$ cases of disease with mild restrictive pattern were found, $2(0.24 \%)$ with moderate restriction and $1(0.12 \%)$ with severe pulmonary restriction.

CONCLUSIONS: The probability of respiratory disease of a restrictive pattern is greater in the mining population. Spirometric values are significantly lower in subjects with occupation in the mining sector.

Keywords: pulmonary function, mining, spirometry.

\section{Introducción}

La minería es una actividad humana que se encarga de explotar la corteza terrestre para sustraer minerales que se hayan formado y acumulado a través del tiempo para su aprovechamiento en toda la industria humana. Esta explotación de los recursos naturales del planeta ha sido siempre un sinónimo de desarrollo (1); por esto mismo, no sorprende que esta actividad sea un catalizador que ayude a la industrialización de las sociedades (2).

Actualmente, el sector minero tiene un alto porcentaje de trabajadores a nivel mundial; sin embargo, sus condiciones laborales están lejos de ser óptimas, presentándose problemas como condiciones precarias de las minas, un bajo salario para los obreros, inestabilidad laboral e incluso tecnología obsoleta (3). A pesar de esto, se han logrado avances en la prevención de riesgos laborales, pero esto está muy lejos de solucionar el problema pues prevalecen en los obreros las afectaciones que van desde eventos traumáticos hasta enfermedades crónicas relacionadas con el ambiente laboral y la exposición e inhalación recurrente de partículas en el aire ambiente dentro de las minas que resultan en enfermedades del sistema respiratorio.

Los trabajos de minería se asocian con el desarrollo de diferentes patologías a nivel osteoarticular, muscular y respiratorio. Una de las enfermedades más comunes en las actividades mineras es la neumoconiosis. Esta enfermedad se presenta mediante el desarrollo progresivo de nódulos redondos en el pulmón, cicatrización y enfisema, aparición de racimos de nódulos amorfos, necrosis, cambios degenerativos vasculares en las arterias bronquiales y pulmonares, lesiones en los vasos linfáticos y liberación de proteínas inflamatorias (4).
Otras enfermedades de gran prevalencia en los trabajadores de minería son la fibrosis masiva progresiva, el cáncer, la enfermedad pulmonar obstructiva crónica (EPOC), la bronquitis crónica y el enfisema pulmonar.

Por una parte, la fibrosis masiva progresiva responde a la formación de cicatrices en gran parte del pulmón (como mínimo de 1,5 cm de diámetro), destrucción del tejido pulmonar y de los vasos sanguíneos de los pulmones (5). Por otra parte, el cáncer de pulmón es la primera causa actual de muerte por cáncer en el mundo. Uno de los principales factores para el desarrollo de cáncer es el cigarrillo; sin embargo, la exposición a arsénico, silicosis y otros minerales están asociados significativamente con esta respuesta celular anómala $(6,7)$.

Debido a que las enfermedades pulmonares en el sector de la explotación minera son recurrentes y afectan significativamente la funcionalidad común y laboral, es importante que los obreros del sector minero se sometan a valoraciones periódicas que permitan identificar el estado de la función pulmonar. De esta manera, una identificación temprana de enfermedad pulmonar permitiría iniciar con un plan de prevención secundaria para evitar y minimizar el riesgo de morbilidad y mortalidad $(8,9)$. En este sentido, la espirometría es una prueba de función respiratoria importante; es un método de valoración de la función pulmonar confiable y reproducible bajo los lineamientos internacionales de la American Thoracic Society (ATS) para su realización, y constituye el estándar de oro para documentar las enfermedades caracterizadas por obstrucción del flujo aéreo (10).

La prueba de espirometría consiste en medir la cantidad de volumen de aire que el paciente puede despla- 
zar durante una espiración forzada en un período breve, cuyo resultado dependerá de ciertos parámetros inherentes de cada individuo que pudieran estar afectados por la exposición a las partículas que se encuentran en el aire de las minas, como el diámetro bronquial y propiedades elásticas del tórax y del tejido pulmonar (11).

Con base en los valores de los resultados de estas pruebas, se podría optar por una intervención temprana, objetiva y asertiva que busque reducir la incidencia de enfermedades respiratorias en la población minera (12). De esta manera, este estudio busca determinar el estado de la función pulmonar en trabajadores de minería mediante espirometría.

\section{Materiales y métodos}

\section{Diseño}

Estudio transversal, descriptivo y observacional con análisis cuantitativo por conveniencia, entre 2016 y 2018 en Cúcuta, Colombia, con 848 participantes trabajadores del sector minero.

\section{Sujetos}

Se contó con una muestra de 848 mineros hombres y mujeres con una edad mayor o igual a 18 años, quienes firmaron previamente un consentimiento informado, el cual permitiría hacer uso de la información necesaria durante el estudio.

Se excluyeron a los sujetos menores de 16 años, sin consentimiento informado o aquellas personas que manifestaran no participar en la investigación. No se tuvieron en cuenta a los sujetos con traqueostomía, problemas bucales o faciales, náuseas incontrolables al introducir la boquilla, incontinencia de esfuerzo, con un estado físico o mental deteriorado, así como también a las personas que tenían muy poco interés en participar, no colaboraban, o no comprendían cómo debía realizarse la maniobra.

\section{Procedimiento}

Los participantes realizaron un cuestionario diseñado por los investigadores de este estudio para recolectar información sobre información personal, carac- terísticas antropométricas y antecedentes personales, patológicos, farmacológicos y tabáquicos.

Previo a la realización de la prueba designada a los pacientes de estudio, se obtuvo la talla y peso de cada uno de ellos. Para hallar el peso, se indicó a los participantes usar la mínima cantidad de ropa durante la medición. Respecto a la talla, los participantes debían estar sin calzado, con el cuerpo estirado y la cabeza en plano de Frankfort; este último es un plano caracterizado por dibujar una línea recta horizontal desde la parte superior del conducto auditivo externo y el borde inferior del ojo a lo largo de ambos lados del cráneo humano y es un hito importante en los estudios de imágenes médicas.

Después de la toma de talla y peso, los participantes debían reposar durante 15 minutos antes de iniciar la prueba y durante ese tiempo se les proporcionaron las indicaciones respectivas sobre la metodología, el objetivo y la disposición para la prueba. Se les explicó cómo debían posicionar la boca, los dientes y la lengua para evitar que se obstruyera el aire.

Cada participante debía mantener una postura erguida sobre el recargo de una silla durante toda la prueba. Así mismo, tendría que mantenerse sin cruzar las piernas y con los pies bien posicionados al suelo. Los pacientes tendrían que respirar a través de la boca, manteniendo al mismo tiempo los ojos cerrados y los labios alrededor de la boquilla para evitar la salida del aire; en algunos casos se utilizaron pinzas nasales para evitar la salida de aire por la nariz.

El instructor de la prueba indicaría a cada participante que realizara una inspiración máxima, lenta, progresiva y debía ser mantenida durante al menos un 1 segundo, y que posteriormente lo expulsase lo más fuerte y rápidamente posible, manteniendo dicha expulsión por 6 segundos. Se corroboró durante la realización de la prueba que no existieran fugas alrededor de la boquilla y se evitaran los cambios de postura del paciente durante la ejecución. Así mismo, se vigilaron los cambios ocurridos durante la prueba, como tos, disnea, dolor torácico y curvas anormales que pudieran ser sospecha de uso de broncodilatadores u otros factores que pudieran modificar el resultado en el desarrollo 
de la prueba. Dicha prueba se repitió hasta 8 veces con la finalidad de conseguir 3 ejecuciones que fueran correctas, según los criterios de la ATS (7).

Para la prueba, se utilizó el espirómetro Spirobank II new. Se determinaron los valores de capacidad vital forzada (FVC), volumen espiratorio máximo forzado al primer segundo $\left(\mathrm{FEV}_{1}\right)$, a los 3 segundos $\left(\mathrm{FEV}_{3}\right)$ y a los 6 segundos $\left(\mathrm{FEV}_{6}\right)$; flujo espiratorio máximo (PEF) Al $25 \%\left(\mathrm{FEF}_{25}\right), 50 \%\left(\mathrm{FEF}_{50}\right), 75 \%\left(\mathrm{FEF}_{75}\right)$ y entre el $25 \%$ y el $75 \%\left(\mathrm{FEF}_{2575}\right)$.

También se determinó el tiempo de espiración forzada (FET), volumen extrapolado (EVol), capacidad vital forzada inspiratoria (FIVC), volumen inspiratorio máximo forzado al primer segundo $\left(\mathrm{FIV}_{1}\right)$, flujo inspiratorio máximo (PIF), edad según la capacidad respiratoria (ELA) y ventilación máxima voluntaria (MVV). Además, se hallaron las relaciones de $\mathrm{FIV}_{1} /$ FIVC, $\mathrm{FEV}_{1} / \mathrm{VC}$ y $\mathrm{FEV}_{3} / \mathrm{FVC}$.

Se contó con una medición de flujo de $\pm 16 \mathrm{~L} / \mathrm{s}$, exactitud del volumen de $\pm 3 \%$ o $50 \mathrm{~mL}$, exactitud del flujo de $\pm 5 \%$ o $200 \mathrm{~mL} / \mathrm{s}$ y con una variación menor de 0,2 litros. La espirometría se realizó en un equipo con neumotacógrafo calefaccionado (Sensormedics), calibrado con una jeringa de 3 litros cada día.

Para la realización de la prueba, se contó con la participación de un especialista en la ejecución de espirometrías y acciones paralelas que hicieron cumplir los mínimos estándares de calidad descritos por la ATS.

\section{Análisis estadístico}

Los datos obtenidos de las variables cuantitativas se analizaron media, mediana, desviación estándar, varianza, asimetría, percentil y coeficiente de correlación de Pearson. Para la comparación de proporciones se utilizaron las pruebas de chi cuadrado $\left(\chi^{2}\right)$ y de McNemar, como también la prueba exacta de Fisher para medidas repetidas.

Para la comparación de medias se utilizó la prueba de la $t$ de Student-Fisher para medidas repetidas o la $t$ de Wilcoxon. El análisis de correlación se determinó mediante la $r$ de Pearson para volúmenes y años de abstinencia de tabaco. Para todas las pruebas, el gra- do de significación estadística elegido fue de 0,05. El análisis de los datos se realizó mediante el programa estadístico IBM SPSS (Statistical Package for the Social Sciences) Statistics, versión 25 para Windows, y el programa Microsoft Excel.

\section{Resultados}

Se tomó una población de 848 sujetos mayores de 18 años con una edad promedio de $31,97 \pm 11,50$ años, sin diagnóstico de enfermedad pulmonar. El 3,30\% (28) de los sujetos en el estudio fueron mujeres y el $96,70 \%$ (820) hombres. Se tomaron medidas de talla, peso e índice de masa corporal (IMC) con valores promedio de: $169,71 \pm 6,77 \mathrm{~cm}, 72,66 \pm 12,56 \mathrm{~kg}$ y 24,35 $\pm 3,57 \%$ respectivamente; el 1,4\% de los sujetos tenía infrapeso (Tabla 1).

Sobre la interpretación de los datos espirométricos, solo $7(0,83 \%)$ casos de la población presentaron EPOC en grado leve y $1(0,12 \%)$ caso tuvo obstrucción pulmonar grave (Tabla 2). En todos los casos, los valores de la función pulmonar en los trabajadores de minería cambiaron significativamente frente a los valores predichos o teóricos (Tabla 3).

Por su parte, aunque aproximadamente el $99 \%$ de la población presentó una relación $\mathrm{FEV}_{1} / \mathrm{FVC} \geq 70$, la diferencia de valores de FVC y FEV 1 de los sujetos y los teóricos alcanzan hasta superar el $80 \%$ del predicho, con un total de $241(28,42 \%)$ casos de enfermedad con patrón restrictivo leve, $2(0,24 \%)$ casos con restricción moderada y $1(0,12 \%)$ caso con restricción pulmonar grave (Tablas 3 y 4 ).

\section{Discusión}

Actividades como la minería aumentan la probabilidad de desarrollar enfermedades respiratorias. Entre algunas de las causas más comunes de enfermedades respiratorias en trabajadores de minas se encuentra la inhalación de gases y minerales como parte del entorno en la explotación de suelos (13).

Este estudio contó con la participación de 848 sujetos trabajadores de minería para la valoración de la función pulmonar a partir de la espirometría, aunque solo $7(0,83 \%)$ casos de la población presentaron 
Tabla 1. Características de los trabajadores de minería

\begin{tabular}{|lccccc|}
\hline & Recuento & Perdidos & Media & Desviación típica & Varianza \\
\hline $\begin{array}{l}\text { Sexo } \\
- \text { M }\end{array}$ & 820 & & & & \\
- F & 28 & & & & \\
Edad (años) & 848 & & 31,97 & 11,50 & 132,29 \\
Talla (cm) & 848 & 169,71 & 6,77 & 45,87 \\
Peso (kg) & 848 & & 72,66 & 12,56 & 157,71 \\
IMC (\%) & 801 & 47 & 24,35 & 3,57 & 12,74 \\
\hline
\end{tabular}

F: femenino; M: masculino.

Tabla 2. Volúmenes, presiones y capacidades pulmonares en hombres y mujeres

\begin{tabular}{|c|c|c|c|c|c|c|}
\hline \multirow{3}{*}{$\begin{array}{l}\text { Volúmenes, } \\
\text { presiones y } \\
\text { capacidades }\end{array}$} & \multicolumn{6}{|c|}{ Sexo } \\
\hline & \multicolumn{3}{|c|}{ M } & \multicolumn{3}{|c|}{$\mathbf{F}$} \\
\hline & Media & Desviación & Varianza & Media & Desviación & Varianza \\
\hline FVC & 4,43 & 0,64 & 0,41 & 3,06 & 0,65 & 0,42 \\
\hline $\mathrm{FEV}_{1}$ & 3,85 & 0,54 & 0,29 & 2,76 & 0,62 & 0,39 \\
\hline PEF & 8,04 & 1,53 & 2,35 & 5,46 & 1,42 & 2,03 \\
\hline FET & 2,41 & 0,88 & 0,77 & 2,05 & 0,57 & 0,33 \\
\hline $\mathrm{FEV}_{1-\mathrm{FVC}}$ & 87,19 & 6,12 & 37,41 & 89,91 & 5,82 & 33,92 \\
\hline $\mathrm{FIV}_{1}$ & 3,54 & 1,30 & 1,69 & 2,29 & 0,97 & 0,94 \\
\hline FIV $_{1}$ FIVC & 3,36 & 1,32 & 1,75 & 2,22 & 0,96 & 0,93 \\
\hline PIF & 86,10 & 29,17 & 850,90 & 86,61 & 31,12 & 968,27 \\
\hline $\mathrm{FEV}_{1} \_\mathrm{VC}$ & 4,24 & 1,97 & 3,88 & 3,00 & 1,50 & 2,24 \\
\hline ELA & 98,40 & 10,99 & 120,67 & 99,94 & 0,25 & 0,06 \\
\hline
\end{tabular}

EPOC en grado leve y $1(0,12 \%)$ caso tuvo obstrucción pulmonar grave.

Annepaka y colaboradores (14) realizaron un estudio con 30 trabajadores de minería de carbón en la población de Khammam, India, no fumadores y aparentemente sanos. Se evaluó la función pulmonar utilizando un espirómetro computarizado con el que se determinaron los valores de $\mathrm{FVC}, \mathrm{FEV}_{1}, \mathrm{FEV}_{1} / \mathrm{FVC} \%$, $\mathrm{FEF}_{2575}$ y PEFR. Los resultados muestran que, para estos valores, disminuyen significativamente en el $100 \%$ de los trabajadores de las minas de carbón frente al predicho o teórico.

En un estudio realizado en Boyacá, Colombia, por Garrote-Wilches y colaboradores (15) con 170 trabajadores masculinos de minería de carbón a quienes se les realizó radiografía de tórax, examen de tuberculina y espirometría, y se determinó que el 15,9 \% de las radiografías de tórax fue compatible con neumoconiosis, y el $17,1 \%$ de las pruebas de tuberculina resultó positivo. El 5,3\% de las espirometrías mostró altera- 
Tabla 3. Valores reales y teóricos $(P)$ de capacidades, volúmenes y presiones pulmonares

\begin{tabular}{|c|c|c|c|c|}
\hline & Media & Cambio (\%) & Desviación & Varianza \\
\hline FVC & 4,38 & \multirow{2}{*}{97,55011} & 0,69 & 0,47 \\
\hline FVC_P & 4,49 & & 0,53 & 0,28 \\
\hline $\mathrm{FEV}_{1}$ & 3,81 & \multirow{2}{*}{101,3298} & 0,58 & 0,33 \\
\hline $\mathrm{FEV}_{1-} \mathrm{P}$ & 3,76 & & 0,46 & 0,21 \\
\hline PEF & 7,96 & \multirow{2}{*}{88,64143} & 1,60 & 2,55 \\
\hline PEF_P & 8,98 & & 0,76 & 0,58 \\
\hline $\mathrm{FEF}_{75-}$ & 2,29 & \multirow{2}{*}{106,0185} & 0,79 & 0,62 \\
\hline $\mathrm{FEF}_{75-} \mathrm{P}$ & 2,16 & & 0,33 & 0,11 \\
\hline $\mathrm{FEF}_{2575}$ & 4,45 & \multirow{2}{*}{100,2252} & 1,05 & 1,11 \\
\hline $\mathrm{FEF}_{2575-} \mathrm{P}$ & 4,44 & & 0,48 & 0,23 \\
\hline FET & 2,40 & \multirow{2}{*}{40} & 0,87 & 0,76 \\
\hline FET_P & 6,00 & & 0,00 & 0,00 \\
\hline $\mathrm{FEV}_{1-} \mathrm{FVC}$ & 87,28 & \multirow{2}{*}{107,8596} & 6,12 & 37,49 \\
\hline $\mathrm{FEV}_{1}$ FVC_P & 80,92 & & 1,76 & 3,11 \\
\hline $\mathrm{FEV}_{6}$ & 4,38 & \multirow{2}{*}{97,55011} & 0,69 & 0,47 \\
\hline $\mathrm{FEV}_{6-} \mathrm{P}$ & 4,49 & & 0,53 & 0,28 \\
\hline $\mathrm{FEV}_{3}$ & 4,36 & \multirow{2}{*}{102,1077} & 0,69 & 0,48 \\
\hline $\mathrm{FEV}_{3-} \mathrm{P}$ & 4,27 & & 0,51 & 0,26 \\
\hline $\mathrm{FEV}_{1-} \mathrm{FEV}_{6}$ & 87,28 & \multirow{2}{*}{104,2274} & 6,12 & 37,46 \\
\hline $\mathrm{FEV}_{1} \mathrm{FEV}_{6-} \mathrm{P}$ & 83,74 & & 1,78 & 3,17 \\
\hline $\mathrm{FEF}_{25}$ & 7,16 & \multirow{2}{*}{92,62613} & 1,46 & 2,12 \\
\hline $\mathrm{FEF}_{25-} \mathrm{P}$ & 7,73 & & 0,60 & 0,36 \\
\hline $\mathrm{FEF}_{50}$ & 4,92 & \multirow{2}{*}{99,19355} & 1,18 & 1,38 \\
\hline $\mathrm{FEF}_{50-} \mathrm{P}$ & 4,96 & & 0,45 & 0,20 \\
\hline $\mathrm{FIV}_{1}$ & 3,50 & \multirow{2}{*}{77,951} & 1,31 & 1,71 \\
\hline $\mathrm{FIV}_{1} \mathrm{P}$ & 4,49 & & 0,53 & 0,28 \\
\hline FIV $_{1}$ FIVC & 3,32 & \multirow{2}{*}{88,29787} & 1,33 & 1,77 \\
\hline FIV $_{1 \_}$FIVC_P & 3,76 & & 0,46 & 0,21 \\
\hline PIF & 86,12 & \multirow{2}{*}{106,4261} & 29,22 & 853,64 \\
\hline PIF_P & 80,92 & & 1,76 & 3,11 \\
\hline $\mathrm{FEV}_{3-} \mathrm{FVC}$ & 80,92 & \multirow{2}{*}{100} & 1,76 & 3,11 \\
\hline $\mathrm{FEV}_{3-} \mathrm{FVC} \_\mathrm{P}$ & 80,92 & & 1,76 & 3,11 \\
\hline ELA & 98,45 & \multirow{2}{*}{103,6098} & 10,81 & 116,76 \\
\hline ELA_P & 95,02 & & 0,57 & 0,33 \\
\hline
\end{tabular}


Tabla 4. Relación entre el FEV y la FVC

\begin{tabular}{|lccccc|}
\hline \multicolumn{1}{|c}{ Relación FEV IFVC $_{1}$} & Media & Desviación & Varianza & Máximo & Mínimo \\
\hline FEV 1 FVC & 87,28 & 6,12 & 37,49 & 100,00 & 68,30 \\
\hline FEV 1 FVC_P & 80,92 & 1,76 & 3,11 & 85,97 & 74,79 \\
\hline FVC & 4,38 & 0,69 & 0,47 & 6,62 & 0,96 \\
\hline FVC_P & 4,49 & 0,53 & 0,28 & 5,95 & 2,05 \\
\hline FEV 1 FEV & 3,81 & 0,58 & 0,33 & 5,56 & 0,80 \\
\hline FE & 3,76 & 0,46 & 0,21 & 4,96 & 1,50 \\
\hline
\end{tabular}

ciones de vía aérea periférica y 2,4 \% mostró un patrón obstructivo.

Otro estudio (16) desarrollado en la población de Jharia Coalfield en la India con un grupo de 170 trabajadores masculinos de minería (66 no fumadores, 52 fumadores y 29 masticadores de tabaco) evaluó la función pulmonar utilizando un espirómetro computarizado con el que se determinó que el 27,64 \% de los trabajadores desarrolló alteraciones pulmonares con un patrón de obstrucción leve, 29,41 \% moderado y $5,88 \%$ grave. En todos los sujetos, los valores de $\mathrm{FEV}_{1} / \mathrm{FVC}$ se encontraron disminuidos significativamente $(65-71 \%$ y $46-53 \%)$.

En el estudio realizado por Oliveira y colaboradores (17) en el que participaron 147 trabajadores masculinos de minería con edad media de 41,37 $\pm 8,71$ años se encontró que $33(22,44 \%)$ trabajadores tenían síntomas respiratorios y 26 (17.69 \%) mostraron anomalías en los resultados de la espirometría. Por su parte, González y colaboradores (3), en su estudio con 226 trabajadores de minas de carbón, encontraron que el $12,3 \%$ (28) de los sujetos presentó enfermedad pulmonar de tipo obstructivo o restrictivo y el $35 \%$ (80) tuvo una disminución de la relación $\mathrm{FEV}_{1} / \mathrm{FVC}$.

\section{Conclusión}

Las pruebas de función pulmonar, como la espirometría, son métodos de evaluación importantes para la identificación de alteraciones pulmonares en trabajadores de minería. Las alteraciones respiratorias son prevalentes en la población minera; sin embargo, la probabilidad de enfermedad respiratoria de patrón restrictivo es mucho mayor frente a la enfermedad obstructiva. Todos los valores espirométricos son significativamente menores en sujetos con ocupación en el sector minero.

\section{Referencias}

1. Castro A, Ronderos C, Bernal F. Minería en Colombia: víctimas, causas y consecuencias del desplazamiento. Ploutos. 2016;5(1), 28-35.

2. Calderón GC. Las problemáticas socio-ambientales generadas por la explotación minera en los páramos de Colombia. Misión Jurídica. 2018;11(13):367-79.

3. González N, Díaz SL, Wilches MR, Franky MP, Méndez C, del Rosario Herrera A. Valoración mediante espirometría de mineros del carbón de Paipa, Colombia. Biomédica. 2017;37(4):498-506.

4. Castranova V, Vallyathan V. Silicosis and coal workers' pneumoconiosis. Environmental health perspectives. 2000;108(4):675-84.

5. Leung $\mathrm{CC}$, Yu IT, Chen W. Silicosis. Lancet. 2012;379(9830):2008-18.

6. Clavero, JM. Estado actual del tratamiento del cáncer pulmonar. Rev Med Clin Condes. 2013;24(4), 611-25.

7. Asociación Latinoamericana de Tórax. Guía Latinoamericana de EPOC - 2014 Basada en Evidencia. ALAT: 2015.

8. López-Giraldo A, Rodríguez-Roisin R, Agustí A. Enfermedad pulmonar obstructiva crónica: la década prodigiosa. Implicaciones para su diagnóstico, prevención y tratamiento. Medicina clínica. 2015;144(11):507-13.

9. Grupo de Trabajo de GesEPOC. Guía de práctica clínica para el diagnóstico y tratamiento de pacientes con enfermedad pulmonar obstructiva crónica (EPOC)-Guía Española de la 
EPOC (GesEPOC). Arch Bronconeumol. 2012;48(1):2-58.

10. Ahern MM, Hendryx M, Conley J, Fedorko E, Ducatman A, Zullig KJ. The association between mountaintop mining and birth defects among live births in central Appalachia, 19962003. Environ Res. 2011;111(6):838-46.

11. Fishwick D, Barber C. Pneumoconiosis. Medicine. 2012;40(6):310-3.

12. Chávez EV, Morales JL, Cortés-Télles A. Espirometría como herramienta en la detección de alteraciones en la función respiratoria. Reporte del Día Mundial de la Espirometría. Evidencia Médica e Investigación en Salud. 2012;5(4):124-30.

13. Díaz JM, Abril FG, Garzón JA. Salud y trabajo: minería artesanal del carbón en Paipa, Colombia. Avances en Enfermería. 2010;28(1):107-15.
14. Annepaka ER, Jhansi K, Amruta K, Rasool S, Munisekhar K. A study on pulmonary function tests in coal mine workers in Khammam District, Telangana-India. Int J Physiother Res. 2014;2(3):502-6.

15. Garrote-Wilches CF, Malagón-Rojas JN, Morgan G, Combariza $\mathrm{D}$, Varona $\mathrm{M}$. Caracterización de las condiciones de salud respiratoria de los trabajadores expuestos a polvo de carbón en minería subterránea en Boyacá, 2013. Rev Univ Ind Sant. 2014;46(3):237-47.

16. Deepa A, Jai KP, Asim KP. Pulmonary Function test of Mine workers exposed to Respirable Dust in Jharia Coalfield India. Int J Scien Eng Res. 2015;6(5):1570-6.

17. Oliveira RCC, Barros JC, Borges F, Andrade M, Veiga PR. Evaluation of pulmonary function and respiratory symptoms in pyrochlore mine workers. J Bras Pneumol. 2016;42(4):279-85. 